Publications of the Astronomical Society of the Pacific, 117:870-884, 2005 August

(C) 2005. The Astronomical Society of the Pacific. All rights reserved. Printed in U.S.A.

\title{
TRISPEC: A Simultaneous Optical and Near-Infrared Imager, Spectrograph, and Polarimeter
}

\author{
Makoto Watanabe, ${ }^{1}$ Hidehiko Nakaya, ${ }^{1}$ Tomoyasu Yamamuro, ${ }^{2}$ Takahiro Zenno, ${ }^{3}$ Miki IshiI, ${ }^{1,4}$ Makoto OKada, \\ Atsushi Yamazaki, Yusuke Yamanaka, Mikio Kurita, Masaru Kino, ${ }^{4}$ Ryuta Ijiri, ${ }^{4}$ Takanori Hirao, \\ Tetsuya Nagata, ${ }^{4,5}$ Shuji Sato, Toshihide Kawai, Youko Nakamura, and Toshikazu Sato \\ Department of Physics, Nagoya University, Furo-cho, Chikusa-ku, Nagoya 464-8602, Japan \\ NoBORU EBIZUKA ${ }^{6}$ \\ Communications Research Laboratory, 4-2-1 Nukui-kita, Koganei, Tokyo 184-8795, Japan \\ AND \\ J. H. Hough and A. Chrysostomou \\ Division of Physics, Astronomy and Mathematics, University of Hertfordshire, College Lane, Hatfield, Hertfordshire AL10 9AB, UK \\ Received 2004 April 6; accepted 2005 May 10; published 2005 July 11
}

\begin{abstract}
We present the design, construction, and performance of TRISPEC (Triple Range Imager and Spectrograph), a simultaneous optical and near-infrared imager, spectrograph, and polarimeter. This instrument splits the incoming light from a telescope into the three beams-one optical channel $(0.45-0.90 \mu \mathrm{m})$ and two infrared channels $(0.90-1.85$ and $1.85-2.5 \mu \mathrm{m})$-by means of two dichroic mirrors. It is capable of simultaneous three-band imaging or spectroscopy, with or without polarimetry. Low-resolution grisms $(R \sim 70-360)$ cover a wide wavelength range of 0.46-2.5 $\mu \mathrm{m}$, simultaneously. The instrument employs three arrays-one $512 \times 512$ SITe CCD and two $256 \times 256$ SBRC InSb arrays-to cover the three channels. It has been commissioned in the imaging, spectroscopic, and polarimetric modes on the United Kingdom Infrared Telescope, the University of Hawaii $2.2 \mathrm{~m}$ telescope, and the Okayama Astrophysical Observatory $1.88 \mathrm{~m}$ telescope since 1999 June.
\end{abstract}

Online material: color figures

\section{INTRODUCTION}

Observations with a wide spectral coverage, including polarimetry, are essential to investigating radiative mechanisms for a number of astronomical objects. Furthermore, simultaneous data collection is crucial to understanding the behavior of a number of objects with short-term variability, such as comets, asteroids, cataclysmic and symbiotic variables, and blazers, many of which exhibit large polarization. Also, simultaneity with a single aperture/slit in an instrument will relieve uncertainties arising from changes in the size and position

\footnotetext{
${ }^{1}$ Current address: Subaru Telescope, National Astronomical Observatory of Japan, 650 North A'ohoku Place, Hilo, HI 96720; watanabe@ naoj.org.

${ }^{2}$ Current address: Genesia Corporation, 3-38-4-601 Shimo-renjaku, Mitaka, Tokyo 181-0013, Japan.

${ }^{3}$ Current address: Purple Mountain Observatory, National Astronomical Observatory of China, 2 West Beijing Road, Nanjing 210008, China.

${ }^{4}$ Visiting Astronomer, Okayama Astrophysical Observatory of the National Astronomical Observatory.

${ }^{5}$ Current address: Department of Astronomy, Kyoto University, Kitashirakawa-Oiwake-cho, Sakyo-ku, Kyoto 606-8502, Japan.

${ }^{6}$ Current address: RIKEN (The Institute of Physical and Chemical Research), Wako, Saitama 351-0198, Japan.
}

of the aperture/slit of separate instruments, as well as changes in atmospheric conditions.

TRISPEC (Triple Range Imager and Spectrograph) is the first "simultaneous" imager and spectrograph with polarimetry covering both optical and near-infrared regions. Other instruments covering both optical and near-infrared regions have been built (Bailey \& Hough 1982; Hough et al. 1991; Epchtein et al. 1994; Nelson et al. 1997; Graham et al. 1998; Kobayashi et al. 1998), but they were limited to simple observational modes, such as photometry and imagery. So far, several simultaneous spectrographs have also been built, such as the Double Spectrograph (Oke \& Gunn 1982) and LRIS (Oke et al. 1995) for the optical, and the Double-Beam Infrared Camera (McLean et al. 1993) and SpeX (Rayner et al. 2003) for the near-infrared. These spectrographs were still limited to either the optical or near-infrared regions.

From the viewpoint of operational efficiency, using a single multiwavelength instrument should always achieve significant gains over using separate instruments. We designed and constructed TRISPEC, a visitor instrument to be used on a variety of telescopes, to work in the optical and near-infrared, delivering gains in operational efficiency and also providing si- 


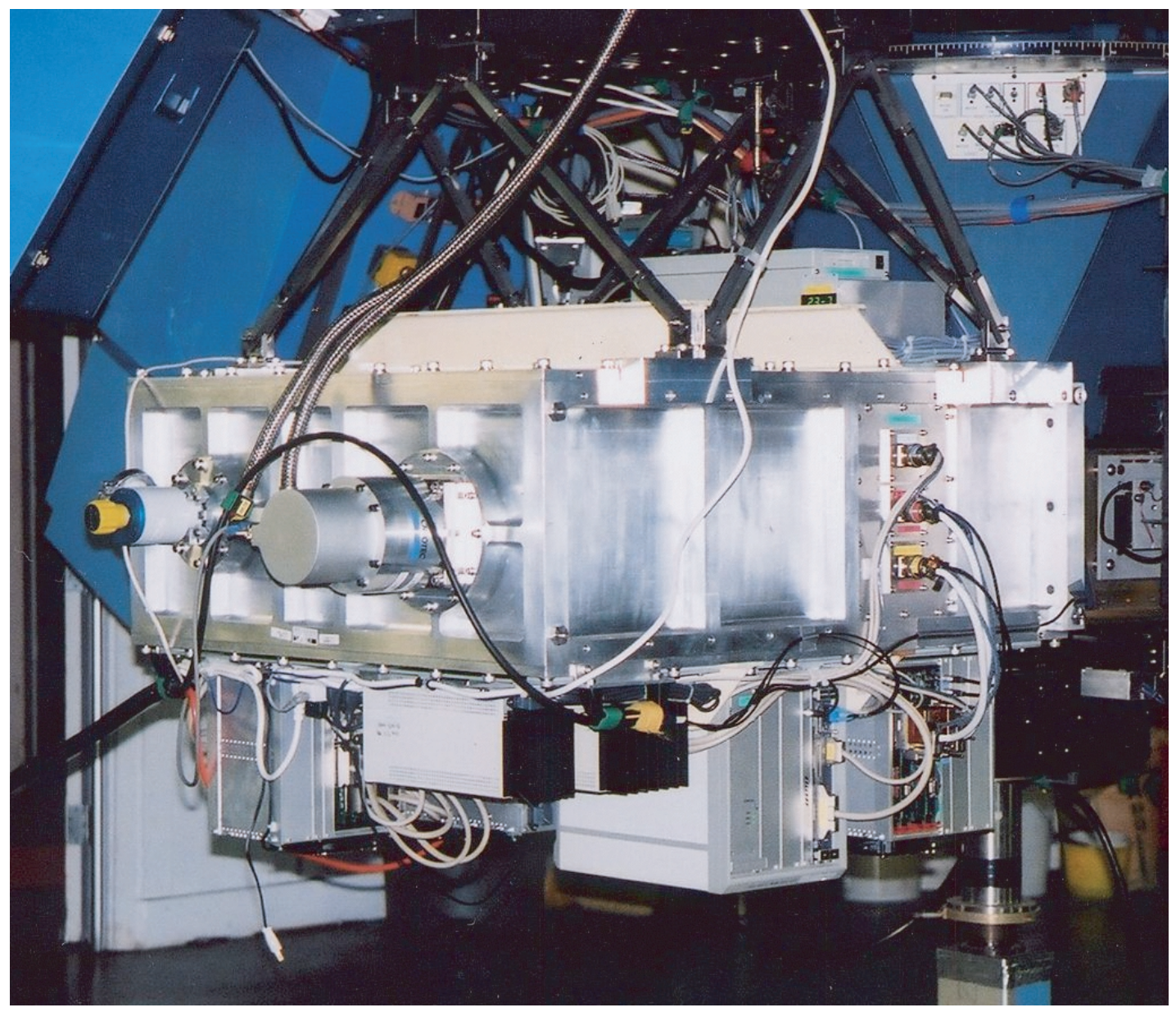

FIG. 1.-Photograph of TRISPEC mounted on UKIRT.

multaneous large-wavelength coverage for variable objects. In TRISPEC, the integration times for normal stars are dominated by the near-infrared observations, because the sensitivity at the $K_{s}$ band is lower than that at the $V$ band by 2 mag. However, there exist numerous interesting objects bright enough in the near-infrared bands but faint or invisible in the optical; i.e., young stellar objects, evolving stars, dust-embedded galactic nuclei, infrared luminous quasars, radio-loud quasars, and so on. TRISPEC is particularly efficient in observing such infrared-bright objects.

In this paper, we describe the development of TRISPEC. We present the design and construction in $\S 2$, the current performance in $\S 3$, and the summary in $\S 4{ }^{7}$

\footnotetext{
${ }^{7}$ Further information on the instrument can be obtained from the TRISPEC Web page at http://www.z.phys.nagoya-u.ac.jp/trispec/.
}

\section{INSTRUMENT DESIGN}

\subsection{Overview}

TRISPEC is a visitor instrument designed to be installed at the Cassegrain foci of various telescopes, including the University of Hawaii (UH) $2.2 \mathrm{~m}$ telescope, the $3.8 \mathrm{~m}$ United Kingdom Infrared Telescope (UKIRT), the Okayama Astrophysical Observatory (OAO) $1.88 \mathrm{~m}$ telescope, and the $8.2 \mathrm{~m}$ Subaru Telescope. Figure 1 shows a photograph of TRISPEC mounted on UKIRT.

The layout of the optics is shown in Figure 2. TRISPEC has two dichroic mirrors splitting the incoming beam into three independent beams: one optical channel $(0.45-0.90 \mu \mathrm{m})$ and two infrared channels (IR1: 0.90-1.85 and IR2: 1.85-2.5 $\mu \mathrm{m}$ ). Each channel has collimator and camera lenses that re-image the telescope's focal plane onto each detector array. The instrument employs one $512 \times 512$ SITe CCD for the optical 


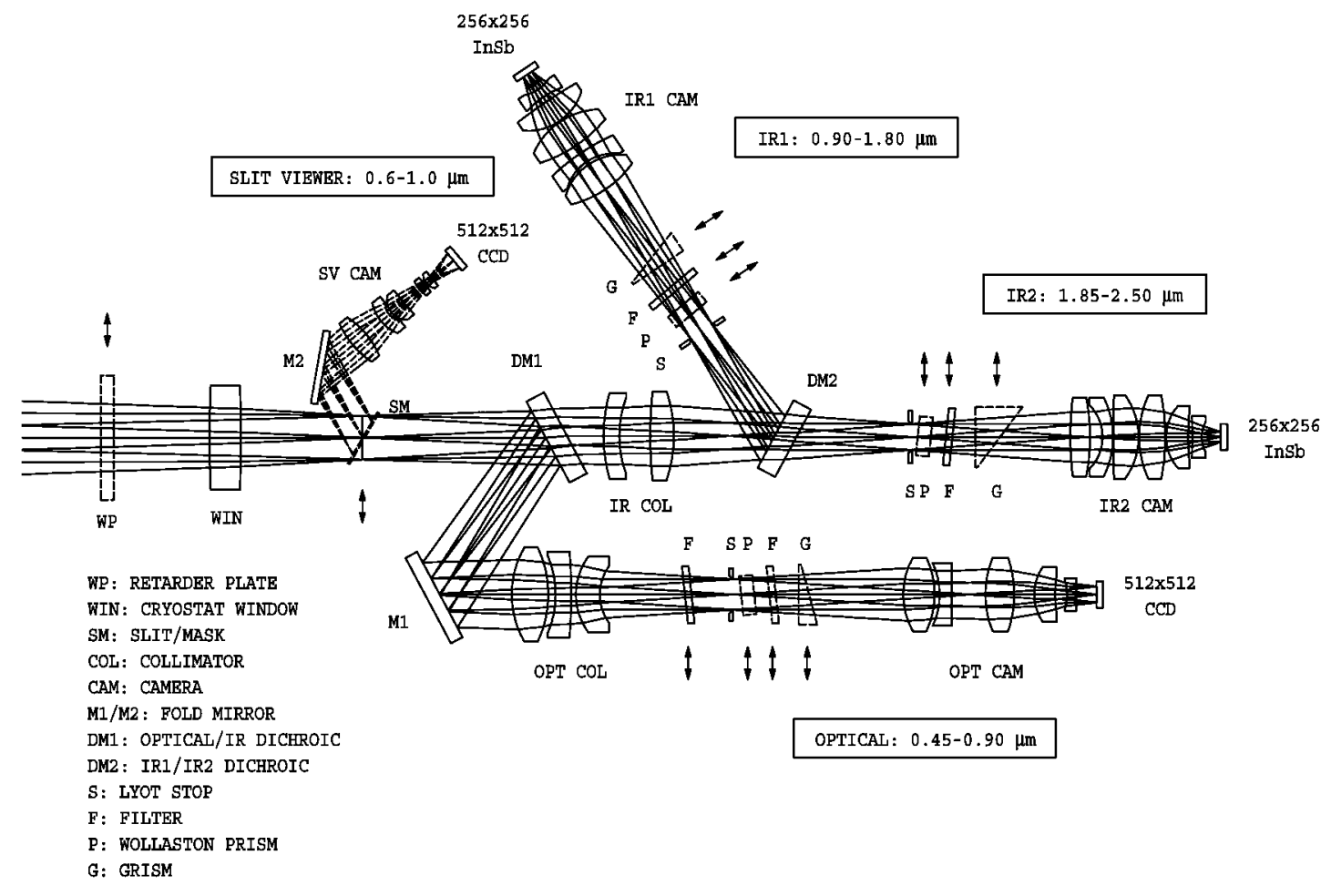

FIG. 2.-Optical layout of TRISPEC.

channel, and two $256 \times 256$ SBRC InSb arrays for the two IR channels. An additional $512 \times 512$ SITe CCD is employed for target acquisition using the field image reflected by a slit mirror.

TRISPEC enables us to realize simultaneous imaging or spectroscopy in the three ranges through a single aperture/slit. It is also capable of polarimetric imaging and spectropolarimetry by inserting Wollaston prisms in front of the filters/ grisms and by rotating wave retarders in front of the cryostat window.

The layout of the cryostat is shown in Figure 3. All the optics, detectors, and mechanisms (wheels and focusing stages) are mounted in the inner cold box, which is supported with glassepoxy plates from the inner wall of the vacuum jacket. The cold box, optics, and mechanisms are cooled down to $\sim 80 \mathrm{~K}$, and the detectors are cooled down to $100 \mathrm{~K}$ for CCDs and to $35 \mathrm{~K}$ for InSb arrays, by a closed-cycle refrigerator. The detector temperatures are regulated within accuracies of $\pm 0.1 \mathrm{~K}$ by a temperature controller. Liquid nitrogen $\left(\mathrm{LN}_{2}\right)$ is also used to boost cooling speed at the beginning of cooling. An iris shutter and a wavelength-calibration unit (WCU) are mounted in front of the cryostat window.

\subsection{Optics}

\subsubsection{Window and Collimator-Camera Optics}

We designed the instrument to have fast final f-ratios ( $\$ 2$ on the Subaru telescope), in favor of observations of low sur- face brightness objects, and we adopted refractive optics because reflective optics with such fast f-ratios were expected to be too large, and the visitor instrument TRISPEC might have lost its portability. However, a number of lenses were required, in particular for the cameras, because of the large distance $(\gtrsim 120 \mathrm{~mm}$ ) between the pupils and cameras for filters and grisms, compared to the focal lengths of the cameras with fast f-ratios.

The focal lengths of the collimator and camera are 262 and $96 \mathrm{~mm}$, respectively, for the optical channel, and 224 and $52 \mathrm{~mm}$ for both of the IR channels. Thus, the combination of the collimator and camera reimages the telescope's focal plane of $33.4 \mathrm{~mm}$ square onto the detector array with a scale reduction of 2.7 to 1 and 4.3 to 1 for the optical and IR channels, respectively, so that the three channels cover nearly the same field of view. The pixel scales and the final f-ratios are listed in Table 1. They are optimized for the Subaru telescope and are 0 .'15 and 0.30 pixel $^{-1}$ with $f / 4.1$ and $f / 2.6$ for the optical and IR channels, respectively. Note that for the UH $2.2 \mathrm{~m}$ telescope with the f/10 secondary, the effective diameter of the telescope is reduced to $1.76 \mathrm{~m}$, because the instrument can accept only an f/12.4 beam. The pupils, or the images of the secondary mirror of the telescope, are formed at the cold stops by the collimators, with the assistance of the slightly curved window of the cryostat. The diameters of the pupils are 23.2 


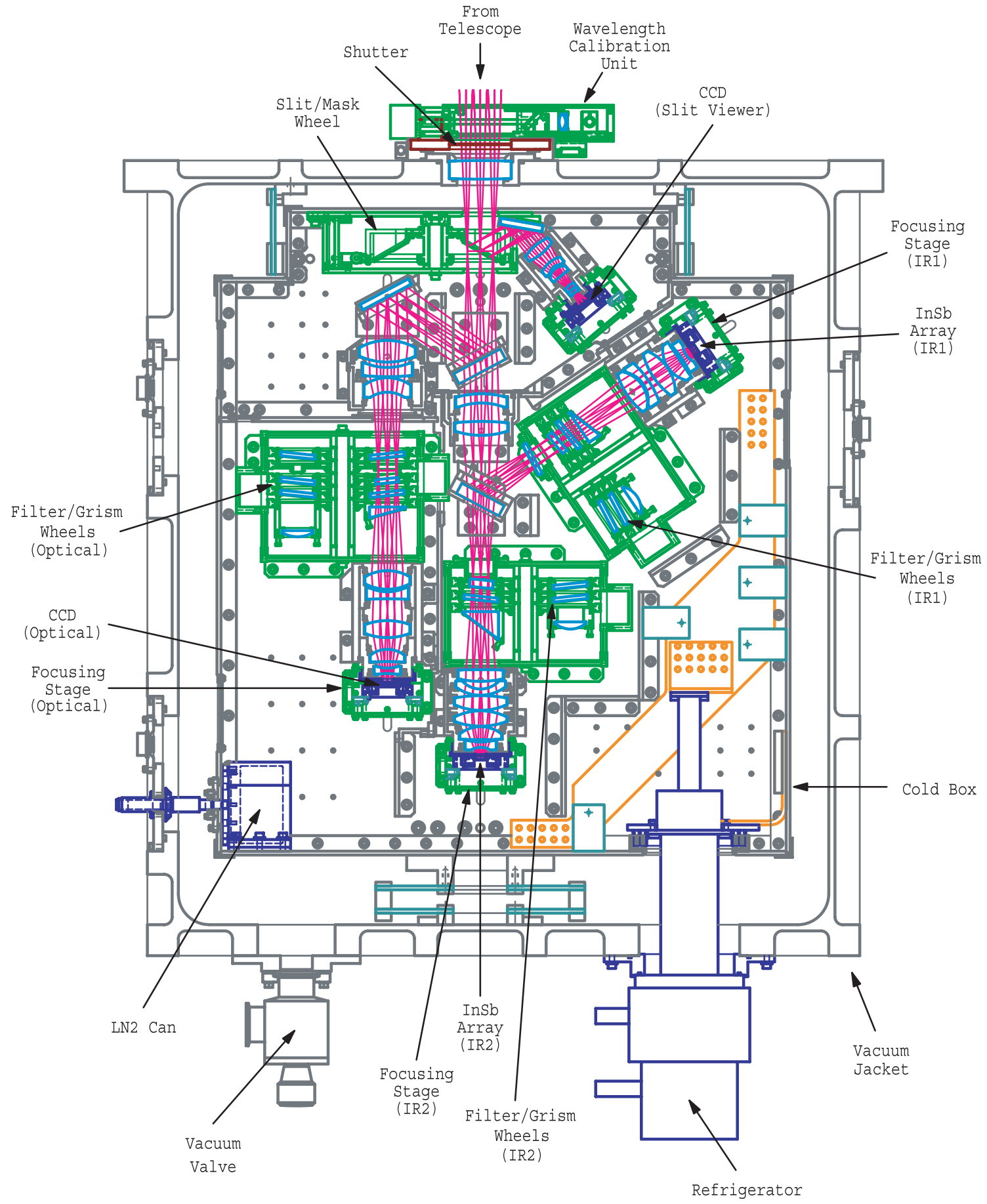

FIG. 3.-Cryostat of TRISPEC. 
TABLE 1

Pixel Scale AND Field of View

\begin{tabular}{|c|c|c|c|c|c|c|c|}
\hline \multirow[b]{2}{*}{ Telescope } & \multirow[b]{2}{*}{ Focus } & \multirow{2}{*}{$\begin{array}{c}\text { SCALE AT } \\
\text { SLIT/MASK } \\
\left(\operatorname{arcsec} \mathrm{mm}^{-1}\right)\end{array}$} & \multirow[b]{2}{*}{$\begin{array}{c}\text { FOV } \\
(\operatorname{arcsec})\end{array}$} & \multicolumn{2}{|c|}{ Optical Channel } & \multicolumn{2}{|c|}{ IR CHANNELS $^{\mathrm{a}}$} \\
\hline & & & & $\begin{array}{l}\text { Final } \\
\text { f-ratio }\end{array}$ & $\begin{array}{c}\text { Pixel Scale } \\
\left(\operatorname{arcsec} \text { pixel }^{-1}\right)\end{array}$ & $\begin{array}{l}\text { Final } \\
\text { f-ratio }\end{array}$ & $\begin{array}{c}\text { Pixel Scale } \\
\left(\operatorname{arcsec}_{\left.\text {pixel }^{-1}\right)}\right.\end{array}$ \\
\hline Subaru & $\mathrm{f} / 12.4$ & 2.3 & 76 & 4.1 & 0.15 & 2.6 & 0.30 \\
\hline UKIRT $\ldots \ldots \ldots \ldots$ & $\mathrm{f} / 36$ & 1.7 & 56 & 12.1 & 0.11 & 7.6 & 0.22 \\
\hline \multirow[t]{2}{*}{$\mathrm{UH} 2.2 \mathrm{~m} \ldots \ldots \ldots$} & $f / 10$ & 10 & 334 & $4.1^{\mathrm{b}}$ & 0.66 & $2.6^{\mathrm{b}}$ & 1.31 \\
\hline & $f / 31$ & 3.3 & 110 & 10.3 & 0.22 & 6.5 & 0.43 \\
\hline OAO $1.88 \mathrm{~m} \ldots \ldots$ & $f / 18$ & 6.5 & 220 & 6.2 & 0.43 & 3.9 & 0.86 \\
\hline
\end{tabular}

${ }^{a}$ The final f-ratio and pixel scale are common between the IR1 and IR2 channels.

${ }^{\mathrm{b}}$ The final f-ratios are limited to 4.1 and 2.6 for the optical and IR channels, respectively, on the UH 2.2 telescope with $\mathrm{f} / 10$ secondary, reducing the effective diameter to $1.76 \mathrm{~m}$.

and $19.9 \mathrm{~mm}$ for the optical and IR channels, respectively, for the $\mathrm{f} / 12.4$ secondary of the Subaru telescope.

In the design phase, the refractive indices of the optical materials were not known at low temperatures, and therefore we measured the refractive indices of 20 materials ( 15 glasses, fused silica, $\mathrm{BaF}_{2}, \mathrm{CaF}_{2}, \mathrm{LiF}$, and KRS-5) at 293, 240, 180, 120 , and $\sim 80 \mathrm{~K}$ at seven wavelengths from 0.36 to $3.3 \mu \mathrm{m}$ (Yamamuro et al. 2005). We used a cryogenic refractometer with a minimum deviation method at Nagoya University. It has a $\mathrm{LN}_{2}$-cooled prism Dewar and uses a heater adjacent to the test prism to obtain temperatures between 293 and $80 \mathrm{~K}$. The changes of the refractive indices with temperature were measured with an accuracy of $\pm 4 \times 10^{-5}$ or better in the refractive index.

We finally chose seven materials for TRISPEC, on the basis of preliminary designs using the results of the refractive index measurements. Table 2 shows the materials and parameters of the lenses in TRISPEC, and Table 3 shows the refractive indices

TABLE 2

Lens PARAMETERS

\begin{tabular}{|c|c|c|c|c|c|}
\hline Material & $\begin{array}{l}\text { Radius of Curvature } \\
\qquad(\mathrm{mm})\end{array}$ & $\begin{array}{l}\text { Distance to Next Surface } \\
\qquad(\mathrm{mm})\end{array}$ & Material & $\begin{array}{l}\text { Radius of Curvature } \\
\text { (mm) }\end{array}$ & $\begin{array}{l}\text { Distance to Next Surface } \\
\qquad(\mathrm{mm})\end{array}$ \\
\hline \multicolumn{3}{|c|}{ Window } & \multicolumn{3}{|c|}{ IR1 Camera } \\
\hline $\mathrm{CaF}_{2} \ldots \ldots \ldots$ & $\begin{array}{r}945 \\
-945\end{array}$ & 24 & $\mathrm{BaF}_{2} \ldots \ldots \ldots \ldots$ & $\begin{array}{c}98.23 \\
-41.518\end{array}$ & $\begin{array}{r}22.917 \\
2.108\end{array}$ \\
\hline \multicolumn{3}{|c|}{ Optical Collimator } & \multirow{2}{*}{$\begin{array}{l}\text { S-TIH14 } \ldots \ldots \ldots \\
\mathrm{BaF}_{2} \ldots \ldots \ldots \ldots \ldots\end{array}$} & -36.456 & 7.987 \\
\hline & $\begin{array}{r}72.802 \\
-90.591\end{array}$ & $\begin{array}{r}27.905 \\
6.830\end{array}$ & & $\begin{array}{c}-128.364 \\
99.64\end{array}$ & $\begin{array}{r}7.761 \\
21.921\end{array}$ \\
\hline S-LAL8 $\ldots \ldots$ & $\begin{array}{r}-77.904 \\
-351.238\end{array}$ & $\begin{array}{r}11.987 \\
1.277\end{array}$ & \multirow{3}{*}{$\begin{array}{l}\text { S-LAL8 } \ldots \ldots \ldots \\
\text { Fused Silica } \ldots \ldots\end{array}$} & $\begin{array}{r}-58.19 \\
40.136\end{array}$ & \\
\hline S-BSL7 $\ldots \ldots$ & $\begin{array}{l}52.044 \\
39.154\end{array}$ & 18.676 & & $\begin{array}{r}3131.552 \\
-108.669\end{array}$ & $\begin{array}{l}3.387 \\
6.999\end{array}$ \\
\hline \multicolumn{3}{|c|}{ Optical Camera } & & 24.008 & \\
\hline $\mathrm{CaF}_{2} \ldots \ldots \ldots$ & 56.288 & 23.918 & \multicolumn{3}{|c|}{ IR2 Camera } \\
\hline S-LAL8 $\ldots \ldots$ & $\begin{array}{l}-63.882 \\
-55.165\end{array}$ & $\begin{array}{l}3.291 \\
8.990\end{array}$ & \multirow{4}{*}{$\begin{array}{l}\mathrm{BaF}_{2} \ldots \ldots \ldots \ldots \ldots \\
\mathrm{LiF} \ldots \ldots \ldots \ldots \ldots \\
\mathrm{CaF}_{2} \ldots \ldots \ldots \ldots \ldots\end{array}$} & $\begin{array}{l}1366.353 \\
-132.97\end{array}$ & $\begin{array}{r}14.946 \\
9.885\end{array}$ \\
\hline & 655.278 & 28.020 & & -32.506 & 7.950 \\
\hline $\mathrm{CaF}_{2} \ldots \ldots \ldots$ & 83.077 & 21.925 & & -53.397 & 0.485 \\
\hline $\mathrm{CaF}_{2} \ldots \ldots \ldots$ & $\begin{array}{r}-80.934 \\
35.084\end{array}$ & $\begin{array}{l}15.014 \\
15.946\end{array}$ & & $\begin{array}{r}188.357 \\
-81.721\end{array}$ & $\begin{array}{r}18.935 \\
0.498\end{array}$ \\
\hline & 295.751 & 8.231 & \multirow{3}{*}{$\begin{array}{l}\mathrm{BaF}_{2} \ldots \ldots \ldots \ldots \ldots \\
\mathrm{BaF}_{2} \ldots \ldots \ldots \ldots \ldots\end{array}$} & 74.843 & 19.928 \\
\hline S-LAL8 & $\begin{array}{r}-46.169 \\
61.043\end{array}$ & 6.992 & & $\begin{array}{r}-96.953 \\
42.508\end{array}$ & $\begin{array}{r}0.498 \\
16.939\end{array}$ \\
\hline \multicolumn{3}{|c|}{ IR Collimator } & & -438.117 & 2.819 \\
\hline & $\begin{array}{r}120.764 \\
83.646\end{array}$ & $\begin{array}{l}11.926 \\
23.667\end{array}$ & \multirow[t]{2}{*}{$\mathrm{LiF} \ldots \ldots \ldots \ldots$} & $\begin{array}{r}-80.14 \\
23.384\end{array}$ & 6.957 \\
\hline $\mathrm{BaF}_{2} \ldots \ldots \ldots$ & $\begin{array}{r}334.691 \\
-108.164\end{array}$ & 17.935 & & & \\
\hline
\end{tabular}

Note. - Parameters for $80 \mathrm{~K}$, except for the window. 
TABLE 3

ReFractive Indices

\begin{tabular}{cccccccc}
\hline \hline $\begin{array}{c}\text { Wavelength } \\
(\mu \mathrm{m})\end{array}$ & $\mathrm{BaF}_{2}$ & $\mathrm{CaF}_{2}$ & $\mathrm{LiF}$ & S-BSL7 & S-LAL8 & S-TIH14 & Fused Silica \\
\hline $0.3651 \ldots \ldots \ldots$ & $\ldots$ & 1.44710 & $\ldots$ & 1.53611 & 1.74574 & $\ldots$ & $\ldots$ \\
$0.435953 \ldots \ldots$ & $\ldots$ & 1.44172 & $\ldots$ & 1.52663 & 1.72957 & $\ldots$ & $\ldots$ \\
$0.54622 \ldots \ldots \ldots$ & 1.47907 & 1.43721 & 1.39586 & 1.51874 & 1.71640 & 1.76938 & 1.45919 \\
$1.014254 \ldots \ldots$ & 1.47170 & 1.43110 & 1.38987 & 1.50741 & 1.69849 & 1.73519 & 1.44937 \\
$1.52999 \ldots \ldots$ & 1.46935 & 1.42840 & 1.38578 & $\ldots$ & 1.68968 & 1.72407 & 1.44343 \\
$2.123 \ldots \ldots \ldots$ & 1.46744 & 1.42549 & 1.38021 & $\ldots$ & $\ldots$ & 1.71390 & $\ldots$ \\
$3.299 \ldots \ldots \ldots$ & 1.46315 & 1.41785 & 1.36432 & $\ldots$ & $\ldots$ & $\ldots$ & $\ldots$ \\
\hline \hline
\end{tabular}

Note. - Absolute values at $80 \mathrm{~K}$ in vacuum.

${ }^{\text {a }}$ Wavelength in vacuum.

at $80 \mathrm{~K}$ adopted for the optical design. The dispersion formulae at room temperature (Browder et al. 1991a, 1991b; Tropf 1995; and the Ohara, Inc., glass catalog) were also used to derive these indices. The indices at wavelengths other than those given in Table 3 were computed from interpolation or extrapolation of the changes of the refractive indices between 293 and $80 \mathrm{~K}$ within the required tolerances $\left( \pm 3 \times 10^{-4}\right.$ in the refractive index). The design was done only for $80 \mathrm{~K}$, and the real temperatures of the optics are indeed close to the designed temperature.

The optics were designed to cover the range $0.36-4.2 \mu \mathrm{m}$ rather than $0.45-2.5 \mu \mathrm{m}$ for a future extension of the spectral coverage. They were designed so that a rms spot size is comparable to a 1 pixel size ( $24 \mu \mathrm{m}$ for $\mathrm{CCD}$ and $30 \mu \mathrm{m}$ for InSb array) throughout the ranges $0.36-0.9,0.9-1.85$, and $1.85-4.2 \mu \mathrm{m}$ for the optical, IR1, and IR2 channels, respectively. Actually, the shortest wavelength, $0.40 \mu \mathrm{m}$ in the optical channel, is limited by the absorption of the lens material, S-LAL8.

\subsubsection{Slit Viewer}

The slit viewer is used to image and guide on an astronomical object at the slit mask. We accepted a limited field of view ( $60 \%$ of that for the three channels) and a poor image quality in the periphery of the field of view, because of the limited space in the cryostat.

Broadband stellar images are subject to elongation due to color aberration by atmospheric dispersion, depending on the zenith angle. We put an R-65 glass filter in front of the siltviewing CCD to define a stellar image between 0.6 and $1 \mu \mathrm{m}$.

\subsubsection{Slits and Aperture Mask}

A slit/mask wheel is located at the telescope focus just behind the cryostat window. The wheel has six positions: three slit mirrors, an aperture mask, and an "open" and a "closed" position. There are two types of slit mirrors: a long-slit and double short-slits, with widths of 300, 450, and $600 \mu \mathrm{m}$, respectively. The double slits are used only for spectropolarimetry, to prevent overlap of the $e$ and $o$ spectra for extended objects. The widths correspond to 4,6 , and 8 pixels for the $\mathrm{CCD}$, and 2, 3, and 4 pixels for the IR arrays. The slit mirrors, polished stainless mirrors with a reflectance of $60 \%-70 \%$, are tilted at an angle of $30^{\circ}$ relative to the incoming beam to reflect the field image to the slit viewer. For imaging polarimetry, an aperture mask with two narrow rectangular apertures is used to avoid overlapping between the $e$ and $o$ images.

\subsubsection{Dichroic Mirrors}

The two dichroic mirrors, DM1 and DM2, split the incoming beam into the three channels by reflecting light of $0.36-0.90$ and $0.90-1.85 \mu \mathrm{m}$, respectively. DM1 and DM2, which were designed and manufactured by Topcon Corp., Japan, are dielectric multilayer coatings on fused silica with a thickness of $15 \mathrm{~mm}$. DM1 has a slight wedge of 0.6 to reduce the astigmatism caused by divergent beams through a parallel plate. The reflectances are above $90 \%$ throughout the range of 0.45 0.90 and $1.0-1.6 \mu \mathrm{m}$ (see $\S 3.2$ for the measured reflectances). The substrates presently limit the sensitivity longward of about $3.5 \mu \mathrm{m}$, because of the absorption. We are planning to replace the substrate with $\mathrm{CaF}_{2}$ for the extension of wavelength coverage to $4.2 \mu \mathrm{m}$.

\subsubsection{Filters}

The optical and both IR channels have three and two filter wheels, respectively, with six holes for each, between the collimator and the camera. For the optical channel, $B, V, R$, and $I$ band glass filters (Bessell 1990) and an order-sorting filter (GG495 glass filter) have been installed. For the IR1 and IR2 channels, $J$ and $H$ band filters and $K, K_{s}$, and $L^{\prime}$ band filters, respectively, provided by the Mauna Kea Filter Consortium (Simons \& Tokunaga 2002; Tokunaga et al. 2002), are installed. Order-sorting 950FH90 (Andover) and Ge filters have been installed for the IR1 and IR2 channels, respectively. Both IR channels also have BK7 glasses to block thermal background radiation, and the IR2 channel has two narrowband filters (3.3 and $3.7 \mu \mathrm{m}$ ). All filters are tilted $6^{\circ}$ relative to the optical axis to eliminate ghosts from the images.

The transmission curves of the glass filters in the optical channel shift by several tens of $\mathrm{nm}$ at low temperatures, causing substantial color changes from the Bessell filters. The color 


\begin{tabular}{|c|c|c|c|}
\hline \multicolumn{4}{|c|}{$\begin{array}{c}\text { TABLE } 4 \\
\text { GRISMS }\end{array}$} \\
\hline Parameter & Optical & IR1 & IR2 \\
\hline Wavelength coverage $(\mu \mathrm{m})$. & $0.46-0.90$ & $0.92-1.80$ & $1.87-2.50$ \\
\hline 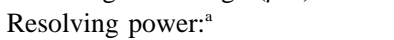 & & & \\
\hline Slit width: $300 \mu \mathrm{m} \ldots \ldots \ldots \ldots$ & 138 & 142 & 360 \\
\hline Slit width: $450 \mu \mathrm{m} \ldots \ldots \ldots \ldots$ & 92 & 95 & 240 \\
\hline Slit width: $600 \mu \mathrm{m} \ldots \ldots \ldots \ldots$ & 69 & 71 & 180 \\
\hline Substrate $\ldots \ldots \ldots \ldots \ldots$ & S-BSL7 & S-BSL7 & S-FTM16 \\
\hline Working order $\ldots \ldots \ldots \ldots$ & 1 & 1 & 1 \\
\hline Blaze wavelength $(\mu \mathrm{m}) \ldots$ & 0.665 & 1.30 & 2.22 \\
\hline Grooves (lines $\mathrm{mm}^{-1}$ ) $\ldots$ & 200 & 120 & 150 \\
\hline Blaze angle $(\mathrm{deg}) \ldots \ldots \ldots \ldots \ldots$ & 15.0 & 17.45 & 36.8 \\
\hline Prism angle $(\mathrm{deg}) \ldots \ldots \ldots \ldots \ldots$ & 15.0 & 18.0 & 36.8 \\
\hline
\end{tabular}

${ }^{\text {a }}$ Resolving power $(\lambda / \Delta \lambda)$ at the blaze wavelength.

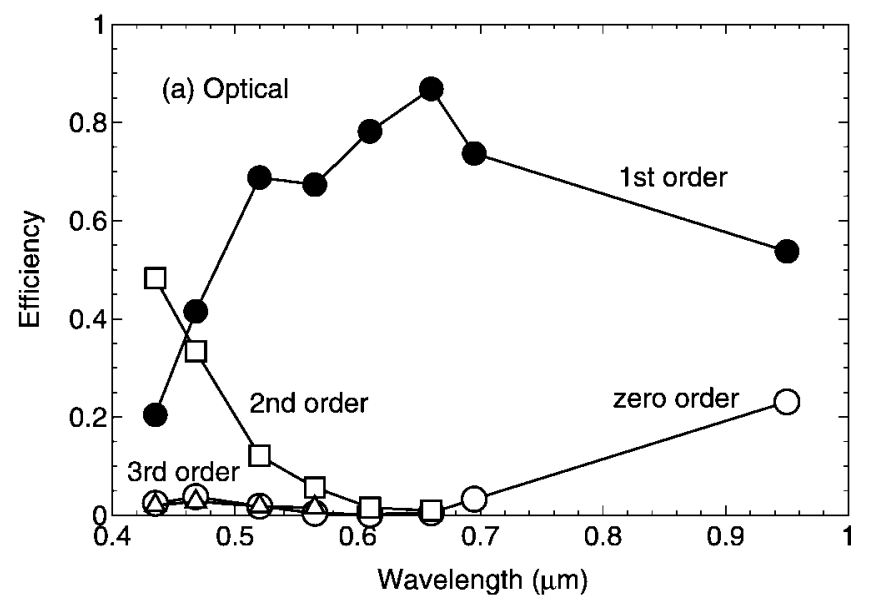

conversion to the Landolt system (Landolt 1992) was determined by Nakaya et al. (2001).

\subsubsection{Grisms}

For spectroscopic observations, each channel is equipped with grisms in the grism wheel located after the filter wheels. Currently, a low-resolution grism with a resolving power of 70-360 is installed for each channel, covering $0.46-0.90,0.92-1.80$, and $1.87-2.5 \mu \mathrm{m}$ simultaneously. All grisms are fabricated by replication of transmission gratings onto glass prisms. The specifications of the three grisms are summarized in Table 4, and the measured efficiencies are shown in Figure 4.

\subsubsection{Wollaston Prisms and Retarders}

For polarimetry, retarders are rotated to set positions in front of TRISPEC, and dual-beam analyzers on the filter wheels for each channel are inserted into the beams. A focal plane mask is used to prevent the overlap of the $e$ and $o$ beams produced

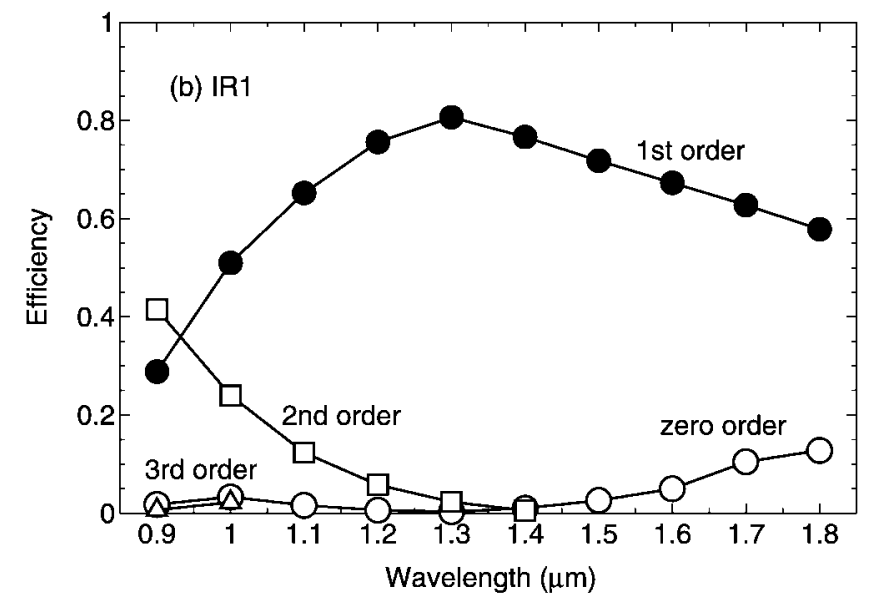

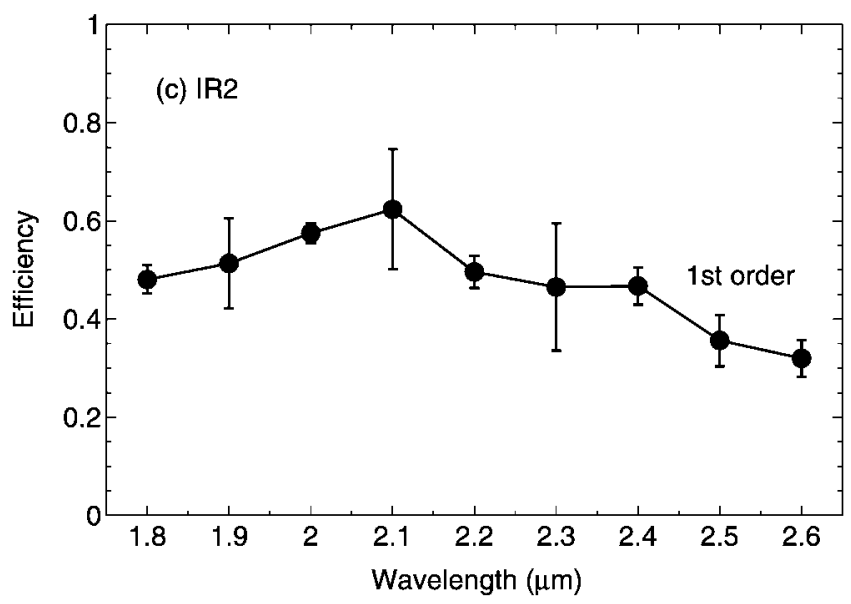

FIG. 4.-Measured efficiencies of the grisms. Panels show the efficiencies of the grisms for $(a)$ optical, $(b)$ IR1, and (c) IR2 channels, respectively. The open and filled circles, rectangles, and triangles indicate the values for the zeroth, first, second, and third order, respectively. 

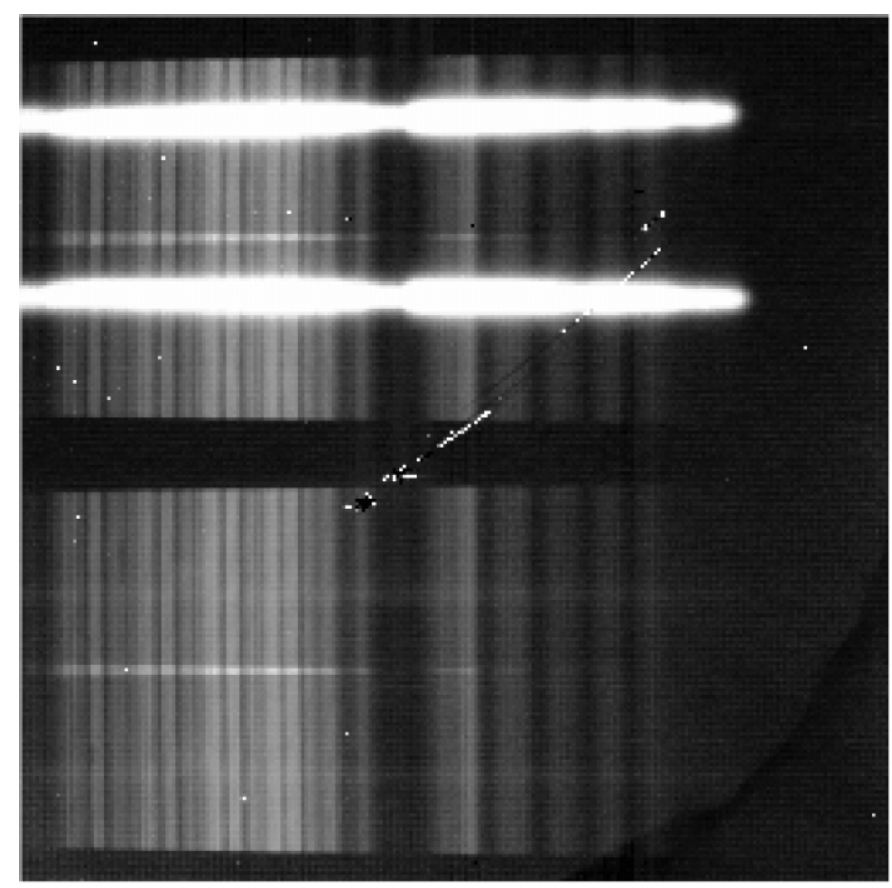

FIG. 5.-Example of raw image in spectropolarimetric mode. Orthogonally polarized ( $e$ and $o$ ray) spectra of HD 38563C (top half) and nearby sky (bottom half) were obtained in the IR1 channel on UKIRT. The horizontal axis corresponds to the dispersion axis, and the right-to-left side of the spectra corresponds to $0.92-1.8 \mu \mathrm{m}$. [See the electronic edition of PASP for a color version of this figure.]

by the analyzer (there are two masks, one for imaging polarimetry and one for spectropolarimetry). Orthogonally polarized images or spectra of both the object and the nearby sky are obtained in each detector array simultaneously, as shown in Figure 5. For linear polarimetry, a single half-wave retarder is used, and for circular polarimetry, the half-wave retarder is spun continuously, followed by a stepped quarter-wave retarder. The continuously rotating half-wave plate reduces the amount of any linear polarization present that is measured as circular, a problem with many polarimeters when only a stepped quarterwave plate is used.
The half- and quarter-wave retarders are Pancharatnam-type superachromats, but were specially designed by B. Halle Nachfl to operate over $0.34-2.5 \mu \mathrm{m}$, compared to the normal superachromats, which are designed for 0.30 to $1.1 \mu \mathrm{m}$ only (Hough \& Aitken 2003). For the half-wave retarder, the retardance varies from 0.48 to $0.52 \lambda$, with a rotation of the fast axis of $\pm 5^{\circ}$ over the full wavelength range. The modulation efficiency remains very high at all wavelengths, and the variation in the fast axis is very easily compensated for by observing a bright star through a calibration polarizer (see $\S 3.6$ for details of the polarimeter performance). Although the retardance of the quarter-wave retarder varies more, ranging from 0.230 to $0.267 \lambda$, and the fast axis rotates by $\pm 3^{\circ}$, the modulation efficiency remains very high. At UKIRT, the retarders are installed in IRPOL2, a facility polarization module. The instrumental polarization is minimized by placing the retarders before any nonsymmetric reflections, such as fold mirrors inside the instrument.

Cemented Wollaston prisms are used as polarization analyzers (see Fig. 6). Ideally, materials should be chosen with both large birefringence, giving large separations of the $e$ and $o$ beams, and low lateral chromatism, giving the minimum image elongation in the polarization dispersion direction. In practice, however, some of the best materials, such as $\alpha$-BBO, are simply not available in the crystal size needed to make the Wollaston prisms (Hough \& Aitken 2003). The separation of polarized spectra is perpendicular to the direction of the spectral dispersion by the grisms. The specifications of the Wollaston prisms are summarized in Table 5.

\subsubsection{Pupil Imaging Lenses}

A plano-convex lens is located in the grism wheel of each channel, and in conjunction with the camera lenses, transfers the pupil image onto the detector array. This function is useful for optical alignment of TRISPEC with the telescope optics.

\subsubsection{Shutter and Wavelength Calibration Unit}

Exposure times for the IR arrays are controlled by the clock sequences from the array controller, while those for the CCDs are determined by a shutter (DC-392, Nidec Copal) with an

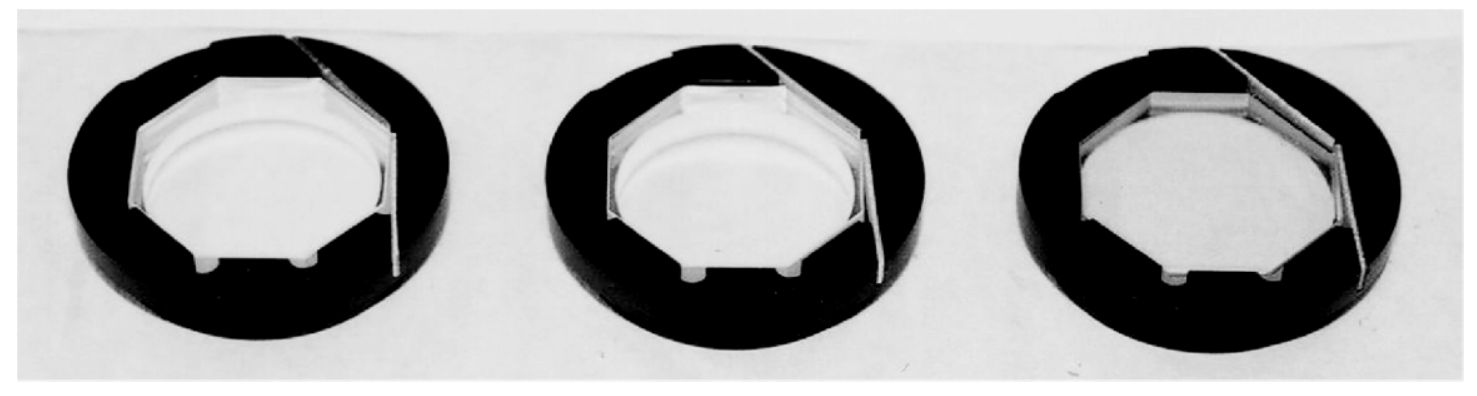

FIG. 6.-Photograph of Wollaston prisms. The left, middle, and right prisms with the holders are for optical, IR1, and IR2 channels, respectively. [See the electronic edition of PASP for a color version of this figure.] 
TABLE 5

WOLLASTON PRISMS

\begin{tabular}{|c|c|c|c|}
\hline Parameter & Optical & IR1 & IR2 \\
\hline Wavelength coverage $(\mu \mathrm{m}) \ldots \ldots \ldots$ & $0.36-0.90$ & $0.90-1.85$ & $1.8-4.2$ \\
\hline Angular separation between $e$ and $o$ beams ${ }^{\mathrm{a}}(\mathrm{deg})$. & $1.61^{\mathrm{b}}$ & $1.76^{\mathrm{c}}$ & $2.23^{\mathrm{d}}$ \\
\hline Pixel separation between $e$ and $o$ beams (pixel) $\ldots$ & $113^{\mathrm{b}}$ & $53^{\mathrm{c}}$ & $68^{\mathrm{d}}$ \\
\hline 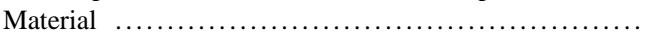 & $\beta$-BBO & $\beta-\mathrm{BBO}$ & $\mathrm{LiNbO}_{3}$ \\
\hline Prism angle $(\mathrm{deg}) \ldots .$. & 6.52 & 7.62 & 15.0 \\
\hline
\end{tabular}

${ }^{\text {a }}$ Derived from the pixel separation, assuming the focal length of the camera to be $96 \mathrm{~mm}$ for the optical channel or $52 \mathrm{~mm}$ for the IR1 and IR2 channels.

${ }^{\mathrm{b}}$ Measured at $0.65 \mu \mathrm{m}$.

c Measured at $1.40 \mu \mathrm{m}$.

${ }^{\mathrm{d}}$ Measured at $2.40 \mu \mathrm{m}$.

opening or closing time of $0.14 \mathrm{~s}$, just in front of the cryostat window.

The WCU contains a Hg-Ar lamp (L937-03, Hamamatsu Photonics) with a condenser lens and is located in front of the shutter. The lamp, mounted on the linear stage, is inserted into the telescope beam to illuminate the slits when wavelength calibration is required.

\subsection{Cryogenics}

\subsubsection{Cryostat}

The vacuum jacket is box-shaped $(1027 \times 920 \times 410 \mathrm{~mm})$. The center section of the vacuum jacket is cut out of a castaluminum block (KN 500, Mitsubishi Chemical). Both the top and bottom covers of the vacuum jacket are $15 \mathrm{~mm}$ thick aluminum plates that are reinforced with two external iron beams and inner aluminum spider bars. The inner wall of the center section is polished to reduce the emissivity.

The cold box is supported by glass-epoxy plates from the front and rear walls of the vacuum jacket and is cooled down by the closed-cycle refrigerator. The cold box is wrapped with triply layered aluminized Mylar sheets to reduce the radiation from the inner wall of the vacuum jacket. The heat inflow is estimated to be $\sim 11 \mathrm{~W}$ as radiation, assuming an emissivity of 0.05 , and $\sim 4 \mathrm{~W}$ as conduction through the glass-epoxy supports.

We employed a two-stage closed-cycle refrigerator (V208C, Daikin Industries) with a capacity of $80 \mathrm{~W}$ at $80 \mathrm{~K}$ for the first stage and $7.4 \mathrm{~W}$ at $20 \mathrm{~K}$ for the second stage. The cold box and the CCDs are cooled with the first stage down to $\sim 80$ and $100 \mathrm{~K}$, respectively, while the InSb arrays are cooled with the second stage down to $35 \mathrm{~K}$. At the beginning of cooling, the cooling speed is boosted by pouring $\mathrm{LN}_{2}$ into a twisted pipe (with a can) attached to the cold box. It takes 24 to $30 \mathrm{hr}$ to cool down from room temperature. For warming up from $80 \mathrm{~K}$ to room temperature, it takes only $\sim 24 \mathrm{hr}$ using 10 heaters (260 W in total) in the cold box.

The inner walls of the cold box and most of the components inside it were painted with "astro-black" polyurethane resin (Nova 500, Nippon Paint) after being sandblasted and/or anodic oxidized. It is robust against thermal cycles, and its reflectance is less than $0.5 \%$ throughout the 0.7 to $4.5 \mu \mathrm{m}$ range, with no spectral features (Ando et al. 2002).

The lens units, mirrors, wheels, and the detectors (mounted on the focusing stages) are lined up along the straight trenches on the bottom plate of the cold box.

\subsubsection{Mechanisms: Wheels and Focusing Stages}

TRISPEC employs 16 cryogenic mechanisms: 12 wheels driven with worm gears and 4 focusing stages driven with screws in the cryostat. All the mechanisms are driven by cryogenic stepper motors. The adoption of internal cold motors made the mechanisms simple and compact and eliminated mechanical failures due to thermal contraction, compared to external warm motors with vacuum feed-throughs.

We employed conventional stepper motors (P430-258-005, Portescap) altered by the replacement of the oiled ball bearings with $\mathrm{MoS}_{2}$-sputtered dry ball bearings (NTN Corp., Japan). These motors were also employed for the Wide Field Cryogenic Telescope (WFCT; Ando et al. 2002) and SIRIUS (Simultaneous Infrared Imager for Unbiased Survey; Nagayama et al. 2003).

The teeth of the brass worm screw were $\mathrm{MoS}_{2}$ coated, while the bushings for the bearings of the wheel and worm shafts were Teflon coated (LF dry bushing, Misumi) to prevent seizing. The focusing stages use Teflon screws to adjust the focus for the detector arrays traveling within $\pm 0.5 \mathrm{~mm}$. Springs apply a constant pressure to the wheels and stages to prevent free movement due to the play of the gears and screws. Mechanical switches sense the zero and/or limit positions of the wheels and stages.

\subsection{Electronics}

A block diagram of the electronics of TRISPEC is shown in Figure 7. The TRISPEC instruments are controlled with a Sun Microsystems workstation located in the control room and a real-time Linux (FSMLabs RTLinux ${ }^{8}$ ) PC mounted on the cryostat. The Sun workstation controls the detector arrays,

\footnotetext{
${ }^{8}$ See http://www.fsmlabs.com.
} 


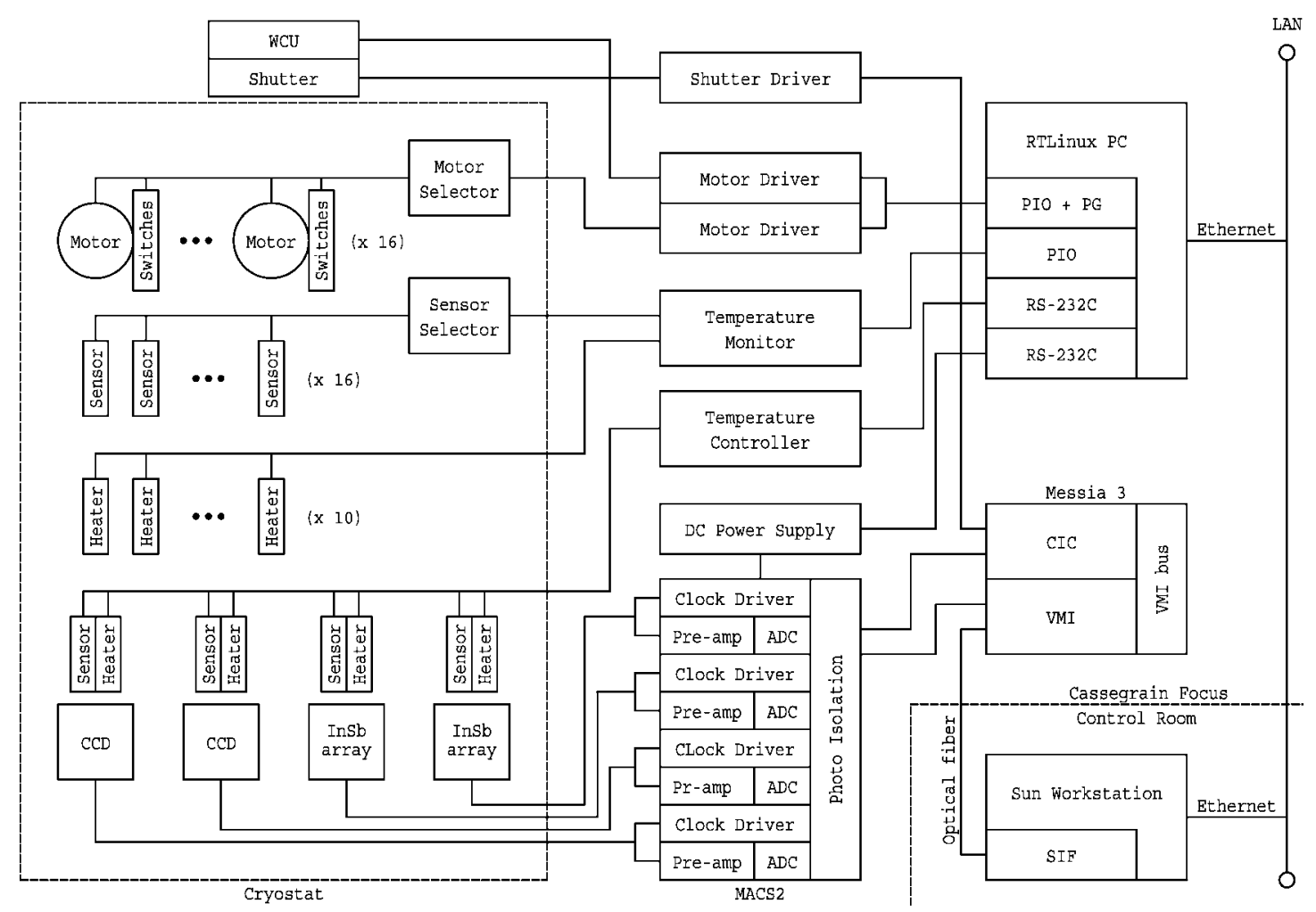

FIG. 7.-Block diagram of TRISPEC control electronics.

while the RTLinux PC controls the mechanisms, including the $\mathrm{WCU}$, and monitors temperatures in the cryostat.

\subsubsection{Array Controller}

We adopted the Messia III (Modularized Extensible System for Image Acquisition; Sekiguchi et al. 1998), which consists of three boards: SIF (Sbus Interface), VMI (VME and Memory Interface), and CIC (CCDs and Instruments Controller). Two digital signal processors (DSPs) on CIC generate clock sequences for arrays and control the shutter. The frame data are stored in VMI and are transferred to the host workstation with SIF through optical fibers.

We adopted MACS2 (Multi-Array Control System; Nakaya \& Sato 1998) as front-end electronics. It consists of (1) an isolation card for isolation from the Messia system, (2) clock driver cards to generate the voltages of DC biases and clocks from CIC, (3) pre-amp cards, and (4) analog-to-digital converter cards. MACS2 is adaptable for both the CCDs and infrared arrays and controls different types of detector arrays simultaneously.

\subsubsection{Motor Controller}

We employed motor selectors in the cold box to reduce the number of motor drivers and to minimize the heat entering the cryostat through the wires. The motor controller, which also turns the WCU lamp on/off, consists of the three sections: (1) a parallel I/O card with a pulse generator, (2) two motor driver cards, and (3) five motor selector cards in the cryostat. The motor selector cards have mechanical relays and analog switches and connect the motor driver card with a pair of the cryogenic motors and the mechanical switches.

\subsubsection{Temperature Controller and Monitor}

TRISPEC has a 4 channel temperature controller for the detectors and a 16 channel temperature monitor for the cold box. The temperature controller regulates temperatures at the four detector arrays within a precision of $0.1 \mathrm{~K}$, monitoring temperatures at eight points around the arrays simultaneously. The temperature monitor senses temperatures at 16 positions distributed throughout the cold box and the vacuum pressure in the cryostat. It also drives the heaters in the cold box during the warm-up.

\subsection{Software}

Exposure times in each channel depend on the spectral energy distribution of objects and the background levels. The optical channel can be exposed for longer periods than both IR channels in most cases, because of the smaller pixel scale, the lower background, and the higher saturation level compared to the IR channels. Therefore, we nest many short IR1 and IR2 
(a) B-band, center

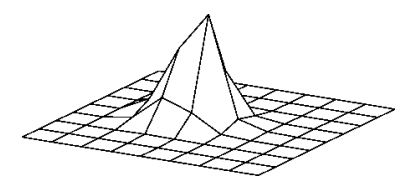

(b) B-band, corner

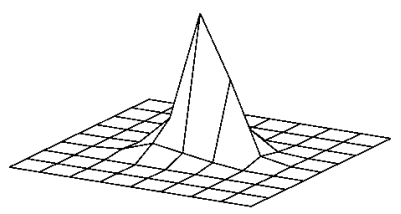

(c) J-band, center

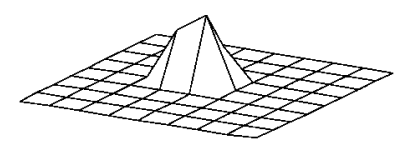

(d) J-band, corner

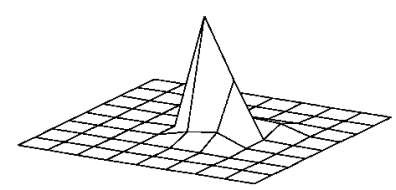

(e) K-band, center

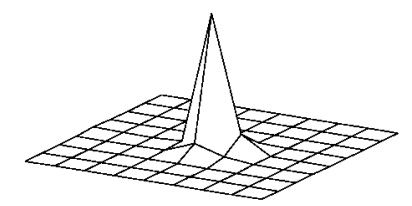

(f) K-band, corner

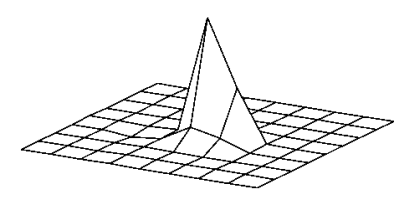

FIG. 8.-Imaging qualities of TRISPEC collimator-camera optics for the $\mathrm{f} / 12.4$ input beam in the laboratory. The figures show profiles of spot images on the detector arrays for pinholes with a diameter of $50 \mu \mathrm{m}$, located at the focal plane behind the window of the cryostat. The pinhole size corresponds to 0.8 pixel for the $B$ band and 0.4 pixel for the $J$ and $K$ bands. Left, middle, and right columns are for the $B, J$, and $K$ bands, respectively. Top and bottom rows show the profiles at the center and corner of the detector arrays, respectively.

exposures within a long optical exposure. The short IR1 and IR2 exposures are also nested within each other or are done simultaneously when both IR exposure times are the same. The numbers of the IR exposures are calculated automatically, and these IR exposures are co-added and averaged for IR1 and IR2 by the software.

To handle many different sequences of exposures for the three channels without changing the DSP program of the Messia system, the command sequence for the DSP is also programmed by the software.

\section{CURRENT PERFORMANCE}

TRISPEC saw first light in the imaging mode in 1999 March and April on the $1.5 \mathrm{~m}$ infrared telescope at Mitaka. It was commissioned in all modes, including spectroscopy and polarimetry, as well as imagery on the UH $2.2 \mathrm{~m}$ telescope and UKIRT on Mauna Kea from 1999 June to 2001 March. After improvements were made to the optics, it was recommissioned on the OAO $1.88 \mathrm{~m}$ telescope in 2003 October and November. We describe the current performance in these runs.

\subsection{Imaging Quality}

As described in $\S 2.2 .1$, the optics were designed so that a spot size of a point source is comparable to a pixel size for each detector array. However, aberrations occurred in all the channels, and in particular, a serious coma appeared in both of the IR channels. In addition, the pupil positions of both IR channels differed from the designed positions; that is, the pupils were obstructed by the stops. In the runs at UKIRT and the UH $2.2 \mathrm{~m}$ telescope with the secondary mirrors set at f/36 and $\mathrm{f} / 31$, respectively, the aberration was negligibly small, owing to the slow f-ratios and the small pixel scales compared to the seeing sizes. Recently, it turned out that the LiF lenses of the IR channels had not been figured correctly, and that some of the lenses in all the channels had not been correctly aligned. Thus, we refurbished the LiF lenses and realigned all the lenses. Figure 8 shows the image qualities after the improvement. The FWHMs of the image spots averaged (for the field of view) are $1.38,1.11$, and 1.02 pixels at the $B, J$, and $K$ bands, respectively, in agreement with the original specifications.

\subsection{Throughput}

We measured the throughput on UKIRT by observing two stars: FS 33 (Hawarden et al. 2001) in the $B, V, H$, and $K$ bands, and CMC 609020 in the $V, J$, and $K_{s}$ bands. In Figure 9 the measured total throughputs are compared to the estimated ones. These include the throughputs of the telescope, dichroic mirrors, lenses, filters, and the quantum efficiencies of the detectors. In both of the IR channels, the measured total throughputs are roughly the same as or somewhat larger than the estimated ones. On the other hand, in the optical channel, the measured throughput is only about half of that predicted, with the difference most likely arising from uncertainties in the throughput of the lenses.

\subsection{Gain, Readout Noise, and Dark Current}

The gains, readout noises, and dark currents of the detectors were measured and are shown in Table 6 . The measured gains were $\sim 10 e^{-} \mathrm{ADU}^{-1}$, in good agreement with the designed specification. The measured readout noises were consistent with the specifications $\left(\sim 9 e^{-}\right.$for the CCD and $<100 e^{-}$for the InSb array; Fowler et al. 1994), using correlated double sampling. 


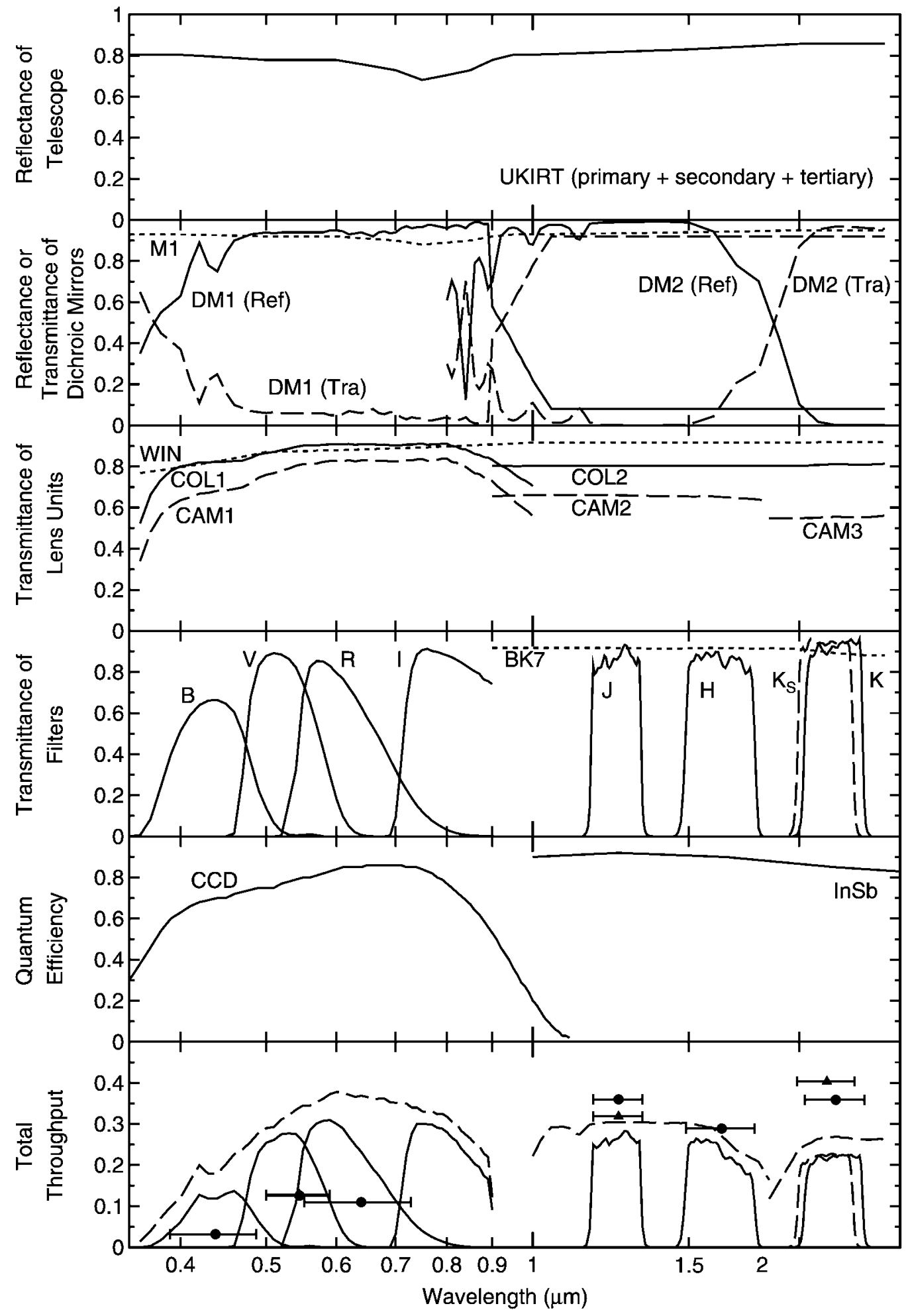

FIG. 9.-Estimated and measured throughputs of TRISPEC on UKIRT. From top to bottom are shown a typical reflectance of telescope, the measured reflectance and transmittance of the dichroic mirrors, the estimated transmittance of the lens units, the measured transmittance of the filters, typical quantum efficiencies of the detectors, and the estimated and measured total throughput, respectively. In the bottom panel, the solid and dashed lines show the estimated throughputs with and without the filters, respectively. The filled circles and triangles show the measured throughputs from the observations of FS 33 and CMC 609020, respectively. 
TABLE 6

Gain, Readout Noise, and Dark Current

\begin{tabular}{cccc}
\hline \hline Parameter & Optical & IR1 & IR2 \\
\hline Detector $\ldots \ldots \ldots \ldots \ldots \ldots$ & SITe CCD & SBRC InSb & SBRC InSb \\
Array format $\ldots \ldots \ldots \ldots \ldots$ & $512 \times 512$ & $256 \times 256$ & $256 \times 256$ \\
Gain $\left(e^{-} \mathrm{ADU}^{-1}\right) \ldots \ldots \ldots \ldots$ & 9.7 & 8.3 & 10.6 \\
Readout noise $\left(e^{-}\right) \ldots \ldots \ldots$ & 18 & 54 & 87 \\
Dark current $\left(e^{-} \mathrm{s}^{-1}\right) \ldots \ldots$ & $\sim 0$ & 3.3 & 39 \\
\hline \hline
\end{tabular}

The dark current for the CCD was undetectable, smaller than $0.1 e^{-} \mathrm{s}^{-1}$ at $200 \mathrm{~K}$. The measured dark current for the IR1 channel was close to the typical value of $1 e^{-} \mathrm{s}^{-1}$, while for the IR2 channel it was quite large compared to the typical value. The latter varies according to changes of temperature in the cold box or room, probably owing to thermal radiation from the components in the cold box or from the wall of the vacuum jacket. Note that the upper quarter of the IR2 array is excluded in this measurement, because of numerous hot pixels.

\subsection{Limiting Magnitude}

The limiting magnitudes were estimated from the measured throughputs, gains, readout noises, and dark currents of the detectors. Table 7 shows the limiting magnitudes for a $3600 \mathrm{~s}$ exposure with four observational modes on UKIRT for a point source. Sky brightnesses of 21.0, 15.9, and $13.7 \mathrm{mag} \mathrm{arcsec}^{-2}$ were assumed in the $V, J$, and $K$ bands, respectively.

\subsection{Spectral Resolution}

The spectral resolutions were confirmed by observing the planetary nebula NGC 7027 on the UH $2.2 \mathrm{~m}$ telescope with the $f / 31$ secondary. In this observation, we employed a long slit of 0 ".36, narrower than the present ones described in $\$ 2.2 .3$. The observed overall spectrum and the spectral line profiles of $\mathrm{H} \alpha(0.6563 \mu \mathrm{m}), \mathrm{P} \beta(1.2818 \mu \mathrm{m})$, and $\mathrm{Br} \gamma(2.1655 \mu \mathrm{m})$ are shown in Figures 10 and 11. These profiles are the instrumental profiles, and their FWHMs agreed well with the expected values of $0.0032,0.0062$, and $0.0042 \mu \mathrm{m}$, corresponding to resolving powers $(\lambda / \Delta \lambda)$ of 206,210 , and 535 at 0.665 , 1.30 , and $2.22 \mu \mathrm{m}$, respectively.

\subsection{Polarization Efficiency and Instrumental Polarization}

We measured the polarization efficiencies by observing a bright star, BS 8845, through a Glan prism inserted in front of the half-wave retarder on UKIRT. We assumed that the Glan prism produces $100 \%$ polarization. The instrument has efficiencies of above $99.5 \%, 97 \%-99 \%$, and $~ 99 \%$ in the optical, IR1, and IR2 channels, respectively (see the top panel of Fig. 12). The unexpected reduction in polarization efficiency of $1 \%-2 \%$ in both IR channels is probably produced by the effect of residual images on the IR arrays.

The bottom panel of the figure shows a change in the position angle of $\sim 20^{\circ}$. The change corresponds to the variation in the orientation of the fast axis for the half-wave retarder by $\sim 10^{\circ}$, in good agreement with the design.

The instrumental polarization was measured by observing unpolarized stars on UKIRT. An example of the measured instrumental polarization is shown in Figure 13. The measured instrumental polarizations were less than $0.1 \%$ in the optical channel and less than $0.3 \%$ in the IR channels.

\subsection{Operating Efficiency}

We measured the operating efficiencies on UKIRT during 11 clear nights between 2001 February 20 and March 2. We defined the operating efficiency as the fraction of total target exposure times while the telescope dome was opened. The averaged operating efficiency was $25 \%, 24 \%$, and $22 \%$ for the optical, IR1, and IR2 channels, respectively. The three channels were mostly exposed simultaneously. Thus, the overall efficiency (the sum of the three) was $72 \%$ during these nights, including several observational modes. Nonexposure time was spent reading out the three arrays and creating image files $(22 \%)$, and working on target acquisition, determining exposure times, filter and mode changes, focusing, etc. (53\%). The integration times were a few seconds in the imaging mode, and longer (several tens or hundreds of seconds) in the spectroscopic modes.

\section{SUMMARY}

We have developed a simultaneous optical and near-infrared imager, spectrograph, and polarimeter, TRISPEC. The instrument covers a wide range, of $0.45-2.5 \mu \mathrm{m}$, with three detector arrays, and is capable of simultaneous three-band imaging and low-resolution spectroscopy, with or without polarimetry. We presented the current performance of TRISPEC. Scientific results have been published by Nakaya et al. (2001), Salyk \& Tholen (2002), and Watanabe et al. (2003).

We thank the staffs of UKIRT, the UH $2.2 \mathrm{~m}$ telescope, and

TABLE 7

Limiting Magnitude

\begin{tabular}{cccc}
\hline \hline Mode & $0.55 \mu \mathrm{m}(V)$ & $1.25 \mu \mathrm{m}(J)$ & $2.20 \mu \mathrm{m}(K)$ \\
\hline Imaging $\left(\mathrm{S} / \mathrm{N}=10,2^{\prime \prime}\right.$ aperture) $\ldots \ldots \ldots \ldots \ldots \ldots$ & 24.4 & 21.8 & 20.4 \\
Imaging polarimetry $\left(\Delta p=0.5 \%, 2^{\prime \prime}\right.$ aperture) $\ldots \ldots \ldots$ & 20.5 & 18.1 & 16.7 \\
Spectroscopy $\left(\mathrm{S} / \mathrm{N}=10,0{ }^{\prime \prime} 88 \mathrm{slit}\right) \ldots \ldots \ldots \ldots \ldots \ldots$ & 21.7 & 20.0 & 17.1 \\
Spectropolarimetry $(\Delta p=0.5 \%, 0.88$ slit $) \ldots \ldots \ldots \ldots$ & 16.9 & 16.2 & 13.4 \\
\hline \hline
\end{tabular}

NoTE. - For $3600 \mathrm{~s}$ exposure on UKIRT. 


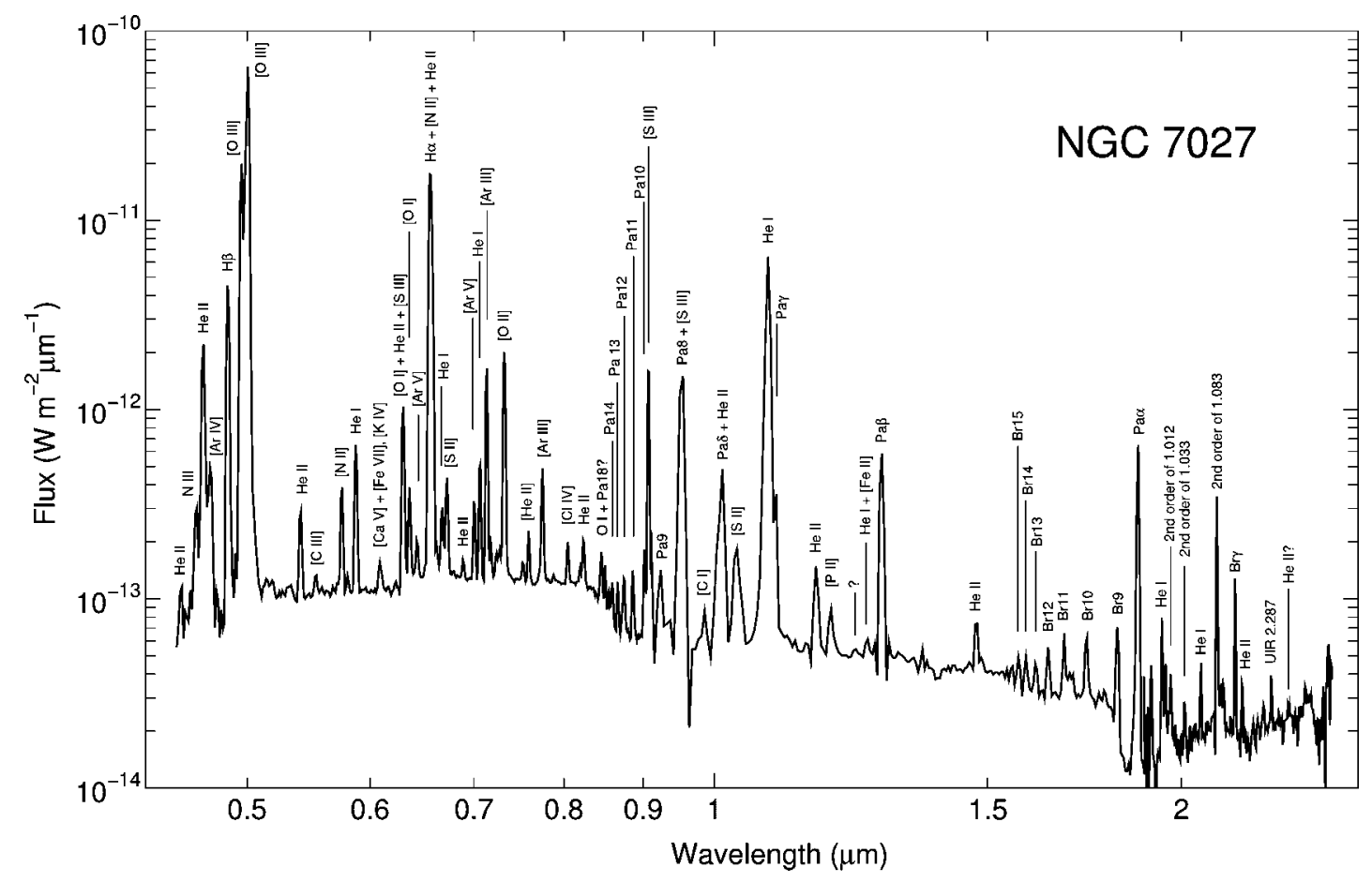

FIG. 10.-Observed spectrum of the planetary nebula NGC 7027 with TRISPEC. The observation was made on the UH $2.2 \mathrm{~m}$ telescope with the f/31 secondary in 1999 September. A narrow slit with a 0"36 width was used, and the resolving powers $(\lambda / \Delta \lambda)$ are thus 206, 210, and 535 at $0.665,1.30$, and $2.22 \mu \mathrm{m}$, respectively. Note that several lines from the second-order light appear around $2 \mu \mathrm{m}$ in the IR 2 channel. Thereafter, the contamination was eliminated by a blocking filter.
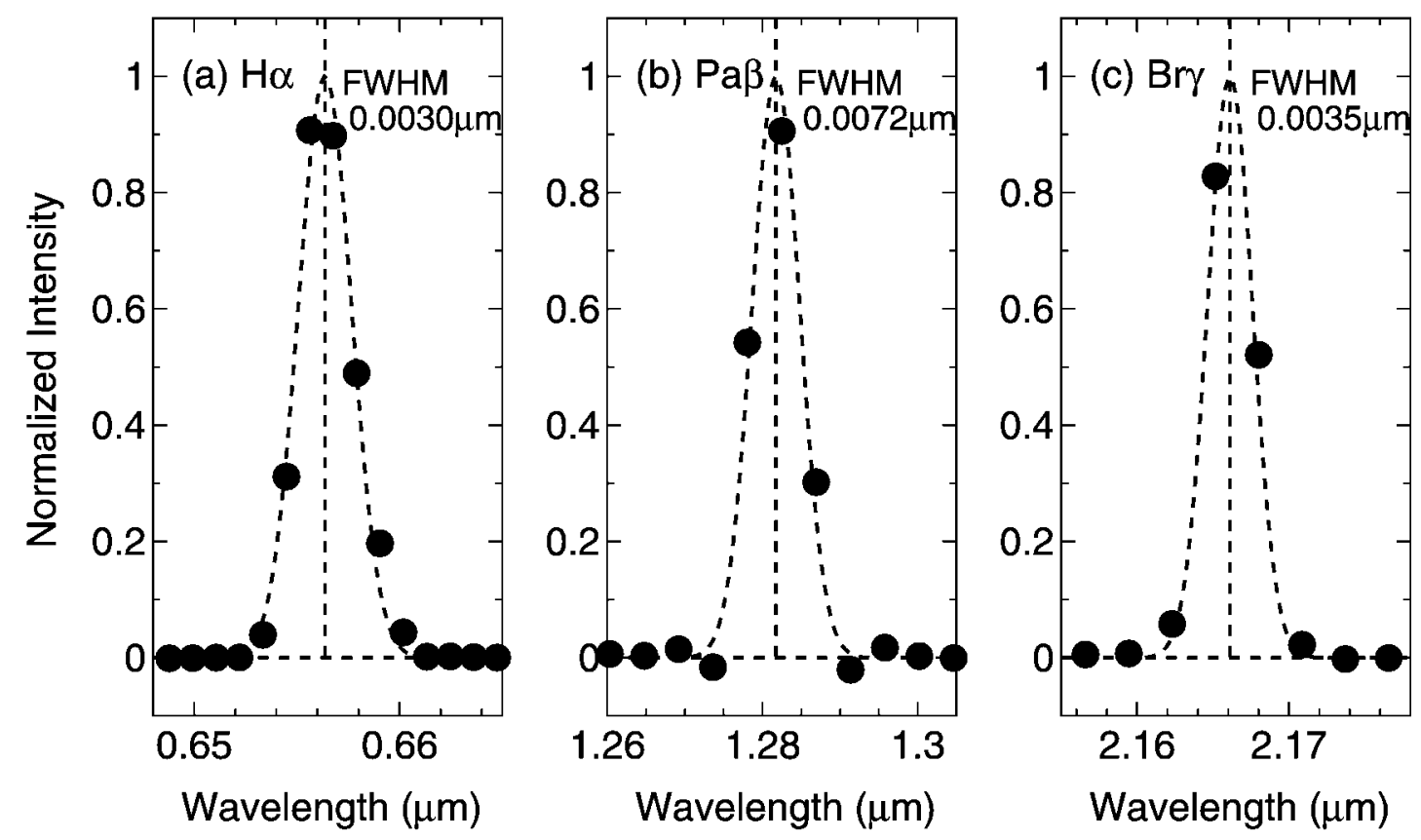

FIG. 11.-Observed spectral profiles of emission lines in NGC 7027. The profiles show (a) $\mathrm{H} \alpha(0.6563 \mu \mathrm{m}),(b) \mathrm{P} \beta(1.2818 \mu \mathrm{m})$, and $(c)$ Br $\gamma(2.1655 \mu \mathrm{m})$. The filled circles show the observational data, and the dashed lines show the Gaussian fitting. The FWHMs from the fitting are shown above the lines. 


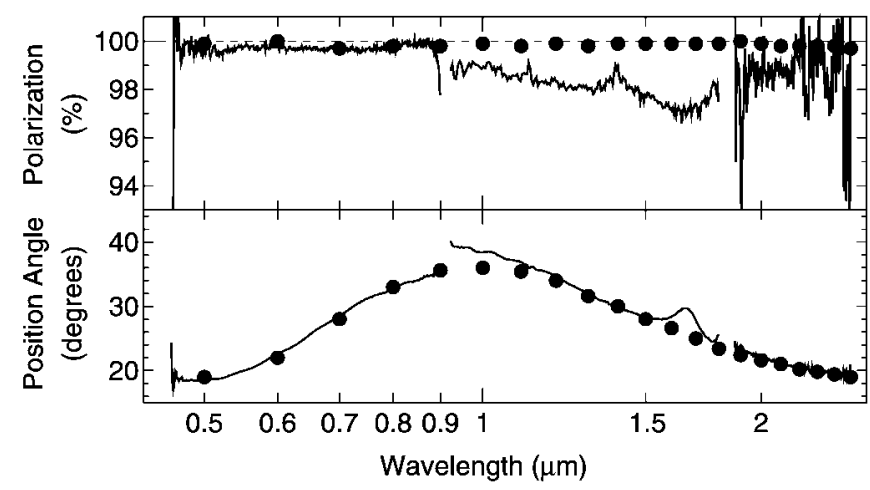

FIG. 12.-Polarization efficiency of TRISPEC by observing the bright star BS 8845 through a Glan prism on UKIRT. The lines present the measured polarization efficiency (top panel) and the change in position angle (bottom panel) corresponding to the variation in the orientation of the fast axis for the half-wave retarder. The filled circles are the design values.

OAO for their support in the observations. We also thank the staff of the Subaru Telescope for help in preparation of the instrument, and Dr. M. Tanaka and the staff of the Advanced Technology Center of NAOJ for their support in the observations at Mitaka. We are grateful to the members of the Z-

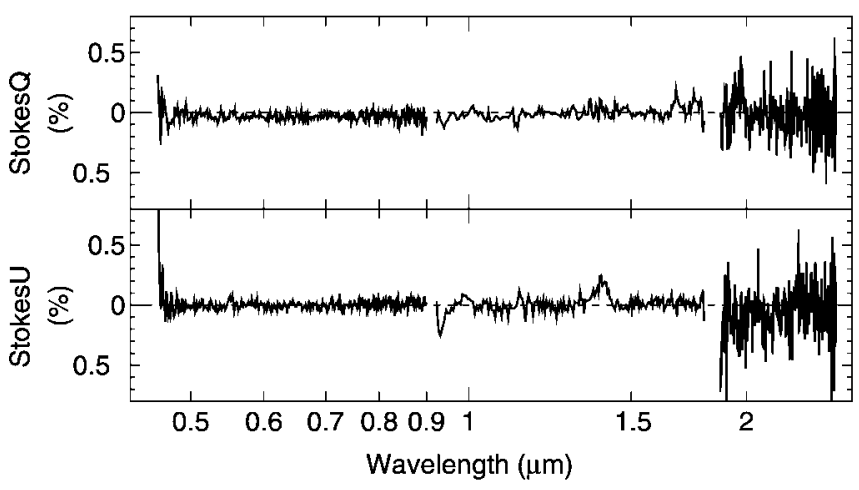

FIG. 13.-Example of measurements of instrumental polarization of TRISPEC. This measurement was made by observing an unpolarized star BS 311 on UKIRT.

Lab, the Department of Physics, Nagoya University, for help in building the instrument. UKIRT is operated by the Joint Astronomy Centre on behalf of the UK Particle Physics and Astronomy Research Council. We thank the Department of Physical Sciences, University of Hertfordshire, for providing IRPOL2 for the UKIRT. This work was financially supported in part by a Grant-in-Aid for Scientific Research from the Ministry of Education, Culture, Sports, Science, and Technology.

\section{REFERENCES}

Ando, M., et al. 2002, Exp. Astron., 13, 119

Bailey, J., \& Hough, J. H. 1982, PASP, 94, 618

Bessell, M. S. 1990, PASP, 102, 1181

Browder, J. S., Ballard, S. S., \& Klocek, P. 1991a, in Handbook of Infrared Optical Materials, ed. P. Klocek (New York: Marcel Dekker), 193

1991b, in Handbook of Infrared Optical Materials, ed. P. Klocek (New York: Marcel Dekker), 427

Epchtein, N., et al. 1994, Ap\&SS, 217, 3

Fowler, A. M., Gatley, I., Vrba, F. J., Ables, H. D., Hoffman, A., \& Wollaway, J. 1994, in Infrared Astronomy with Arrays, ed. I. S. McLean (Dordrecht: Kluwer), 399

Graham, J. R., Hillenbrand, L. A., \& Misch, A. A. 1998, PASP, 110, 732

Hawarden, T. G., Leggett, S. K., Letawsky, M. B., Ballantyne, D. R., \& Casali, M. M. 2001, MNRAS, 325, 563

Hough, J. H., \& Aitken, D. K. 2003, Proc. SPIE, 4843, 200

Hough, J. H., Peacock, T., \& Bailey, A. 1991, MNRAS, 248, 74

Kobayashi, Y., et al. 1998, Proc. SPIE, 3354, 769

Landolt, A. U. 1992, AJ, 104, 340

McLean, I. S., et al. 1993, Proc. SPIE, 1946, 513
Nagayama, T., et al., 2003, Proc. SPIE, 4841, 459

Nakaya, H., \& Sato, S. 1998, Proc. SPIE, 3354, 368

Nakaya, H., Watanabe, M., Ando, M., Nagata, T., \& Sato, S. 2001, AJ, 122, 876

Nelson, B., McLean, I. S., Henriquez, F., \& Magnone, N. 1997, PASP, 109,600

Oke, J. B. \& Gunn, J. E. 1982, PASP, 94, 586

Oke, J. B., et al. 1995, PASP, 107, 375

Rayner, J. T., et al. 2003, PASP, 115, 362

Salyk, C. \& Tholen, D. J. 2002, BAAS, 34, 1406

Sekiguchi, M., Nakaya, H., Kataza, H., \& Miyazaki, S 1998, Exp. Astron., 8, 51

Simons, D. A., \& Tokunaga, A. T. 2002, PASP, 114, 169

Tokunaga, A. T., Simons, D. A., \& Vacca, W. D. 2002, PASP, 114, 180

Tropf, W. J. 1995, Opt. Eng., 34, 1369

Watanabe, M., Nagata, T., Sato, S., Nakaya, H., \& Hough, J. H. 2003, ApJ, 591, 714

Yamamuro, T., Sato, S., Zenno, T., Takeyama, N., Matsuhara, H., Maeda, I., \& Matsueda, Y. 2005, Appl. Opt., submitted 